\title{
MÉDITATIONS D'ARRIÈRE-SAISON. FRANZ LISZT À TIVOLI
}

\author{
PIERRE MORABIA \\ Pianiste \\ p.morabia@free.fr
}

\begin{abstract}
Liszt a vécu plusieurs vies. Pierre Morabia s'intéresse à ses séjours italiens à la Villa d'Este, qui coïncident avec sa maturité de compositeur. C'est l'occasion, pour le pianiste, d'analyser trois pièces du troisième recueil des Années de pèlerinage (1877), qui précèdent les compositions à caractère initiatique des dernières années, mais recèlent des trésors d'expressivité dont le but est beaucoup plus la vérité de l'artiste sincère que l'artifice du virtuose.
\end{abstract}

Mots-clefs : Franz Liszt, Villa d'Este, Tivoli

La Villa d'Este, bâtie au milieu du XVI e siècle par le cardinal Hippolyte II d'Este au sommet des pentes occidentales de la petite ville de Tivoli, à une trentaine de kilomètres à l'est de Rome, reçoit chaque année de très nombreux visiteurs, attirés par son architecture raffinée aux élégants décors maniéristes, par sa situation magnifique, entourée de jardins somptueux qui dévalent la colline, agrémentés de jeux d'eau ruisselant sur les pentes où les bassins, les fontaines et les statues composent un décor inoubliable. Un lieu où la nature, l'histoire et la poésie se rejoignent. Ce faisant ils suivent les pas d'autres visiteurs célèbres qui ont arpenté ces lieux depuis bientôt cinq siècles : outre quelques papes, Benvenuto Cellini, Titien, le Tasse ou encore Montaigne, Fragonard, Hubert Robert... Chez aucun cependant, elle n'aura laissé une marque aussi profonde que chez Franz Liszt, dont elle fut presque une seconde maison durant les vingt dernières années de sa vie ( ce que signale d'ailleurs une inscription bilingue italien-hongrois à l'entrée de l'édifice, accompagnée d'un médaillon au profil du musicien).

Il s'y plaisait beaucoup, l'appelait même son « Eldorado », et y fit des séjours fréquents à partir de 1866, à l'invitation du cardinal Hohenlohe, lors de ses venues à Rome. L'on sait qu'à partir de 1869, la vie de Liszt, « trifurquée » selon sa propre expression, se partage chaque année entre Budapest (où sous son impulsion se développe l'Académie de Musique nouvellement fondée, dont les locaux initiaux jouxtent son appartement de la Andrássy út, actuel Musée Liszt), Weimar (où l'attendent de nombreux élèves venus de l'Europe entière et une vie musicale et mondaine intense qu'il a longtemps animée) et Rome (chère à son cœur depuis la retraite de deux ans effectuée au cloître de la Madonna del Rosario à l'issue 
de laquelle il reçut les ordres mineurs franciscains, et où il a noué de nombreuses amitiés).

Mais, à l'écart de la grande ville, il trouve à la Villa d'Este un cadre propice au travail et à la méditation, dans le silence et la sérénité. Très tôt levé, il entend la messe de bon matin, avant de s'atteler à la tâche, en travailleur acharné qu'il a toujours été : composition, correspondance, à l'occasion quelque visiteur à recevoir ou quelque leçon à donner.

Ce cadre enchanteur nous vaudra les trois dernières grandes pièces du piano lisztien, écrites neuf ans avant sa mort, nettement antérieures aux ultimes pages, énigmatiques, parfois, voire expérimentales, et passionnantes dans leur étrangeté, mais jamais très développées. Ces trois pièces forment le cœur du troisième recueil des Années de Pèlerinage, postérieur de plusieurs décennies aux deux premiers. Méditations d'arrière saison d'un musicien précocement vieilli chez qui « l'amertume du cœur » a succédé à « l'allégresse du cœur » et qui, au terme d'une vie plus que remplie («c'était bien assez de la vivre ma vie »), cherche le réconfort dans sa foi.

Au printemps 1877, il est atteint des premiers accès de profonde mélancolie qui jalonneront ses vieux jours. «Parfois la tristesse couvre toute mon âme comme d'un linceul. » De retour à Tivoli, à la fin de l'été, il écrit le 23 septembre à la princesse Wittgenstein : "Ces trois jours, je les ai passés tout entier sous les cyprès. C'était une obsession, impossible de songer à autre chose, même à l'église. Les vieux troncs me hantaient, et j'entendais chanter et pleurer leurs rameaux chargés de leur inchangeable feuillage ! Enfin les voilà couchés sur du papier à musique... »

Ainsi naquirent les deux pièces intitulées « Aux cyprès de la Villa d'Este ». Nulle intention descriptive derrière ce titre, il ne s'agit pas de peindre la majesté des grands arbres, ni le vent dans les branchages, mais d'évoquer les états d'âme du poète devant ces «tristes compagnons », dressés vers le ciel tels des stèles immuables. Quelques jours plus tard, il précise : «Depuis une quinzaine de jours je suis tout en cyprès (...). J'ai donc composé deux groupes de cyprès, chacun de plus de deux cents mesures, plus un postludium, aux cyprès de la Villa d'Este. Ces tristes choses n'auront guère de succès, et s'en passent. Je les intitulerai thrénodies, le mot d'élégie me paraissant trop doux et quasi mondain. » Ce double titre explicite le caractère funèbre de ces sombres méditations, incarné dans une langue intensément chromatique, en permanente instabilité tonale. La mort, qui a souvent occupé ses pensées est là bien présente. N'a-t-il pas confié au cardinal Hohenlohe, son hôte : « Si j'avais le choix de ma sépulture, ce serait au cimetière de Tivoli »? (Il ne sera pas exaucé...).

C'est bien dans un climat oppressant que débute la première pièce Andante. Sur le lourd balancement octavié des basses, tel une pesante pulsation cardiaque, s'amorce un thème d'accords où se dessine déjà la cellule mélodique $a$ 

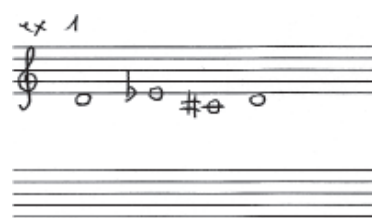

motif serré, noué autour de ses demi-tons, sorte de B. A. C. H. anxieux, qui innervera le morceau entier. Tout ce début baigne dans l'incertitude tonale, il s'agit en fait d'une longue dominante, imprégnée de l'accord instable de quinte augmentée, en mode « hongrois » à deux notes sensibles, do\# et fa\#, brodée et appoggiaturée, qui ne se résout qu'à l'arrivée du chant molto accentuato en sol mineur (mes. 33). Tonalité à peine touchée que l'on quitte déjà, emporté par le premier élan mélodique. Cette longue phrase, prenant appui sur $a$, irrésistiblement ascendante, pendant que les basses s'enfoncent chromatiquement, " mélodie quasiment amoureuse » (dixit Liszt), s'élance, éperdue, vers un premier sommet expressif, empruntant au passage les tons les plus éloignés, fa\# mineur, la mineur, mib mineur, solb majeur, pour lentement retomber tranquillo, mes. 33, selon un jeu d'oppositions ombre/lumière typiquement lisztien. Retour, sur des batteries chuchotées en croches, au chant initial, désolé (oscillations de sol mineur et de fa\# mineur, mes. 63-66, puis de si mineur et de la\# mineur, mes. 71-74).

$\mathrm{S}$ 'amorce alors la progression énorme, presque effrayante, qui va constituer le cœur du morceau : glissement chromatique ascendant de $a$ (mes. 79-86), qui $s$ 'ancre sur le motif pédale $a$ en octaves à la basse autour de do\#, pour finalement prendre feu en accords alternés aux deux mains et (toujours sur cette pédale de do\# en trémolos caverneux) exploser en un cri expressionniste (rinforzando, mes. 116), mais vain (tout n'est que vanité, dit l'Ecclésiaste, même les plus grands élans), s'épuisant sans issue autour de l'intervalle diabolus in musica de quarte augmentée.

Rappel tronqué de l'élan éperdu initial et voilà, miraculeusement amenée, l'éclaircie de la coda, mes. 147, « vers la fin un rayon de teint religieux l'éclaire » (lettre du 27 septembre 1877). Le motif $a$ transformé en

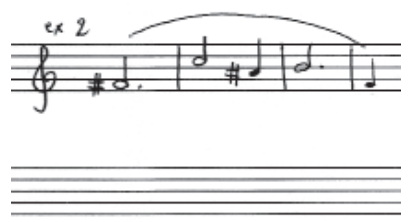

pur, se dénoue à travers un épilogue en questions-réponses, dorénavant diatonique, apaisé jusque dans ses élans passionnés (mes. 168-171ff, à comparer à 131-134). Toutes les tensions semblent fondre sur les chromatismes descendants 
(mes. 176 et suivantes) qui inversent le motif initial, pour s'évaporer dans de longues résonances arpégées... Certitude des derniers accords.

« Le renoncement à toute chose terrestre fut l'unique mobile, le seul mot de ma vie. » Renoncement douloureux néanmoins, en témoigne ce morceau violent, tendu, né d'impulsions véhémentes, dont on ne sort pas indemne et qui projette en nous ses interrogations profondes.

La seconde pièce, andante non troppo, thrénodie également, est pourtant assez différente. Ici, à l'inverse des flux et reflux en constante évolution du premier morceau monothématique, des séquences très caractérisées et clairement manifestées par les changements d'armure (mi mineur, sib majeur, fa\# majeur...) vont être abruptement juxtaposées les unes aux autres, en prenant valeur de symbole, tel des leitmotiv, comme autant d'états d'âme d'un vieil homme faisant retour sur son existence.

L'entrée est saisissante : un motif intensément chromatique dont les dissonances violentes se résolvent dans un souffle, thème de la souffrance et du désir au climat tristanesque évident, si non citation textuelle.

On a relevé chez Wagner une quarantaine d'emprunts directs à Liszt. Ici le jeu d'échanges croisés remonte loin. De 1840 date le Lied composé par Liszt, intitulé Die Lorelei, sur le fameux poème de Heine

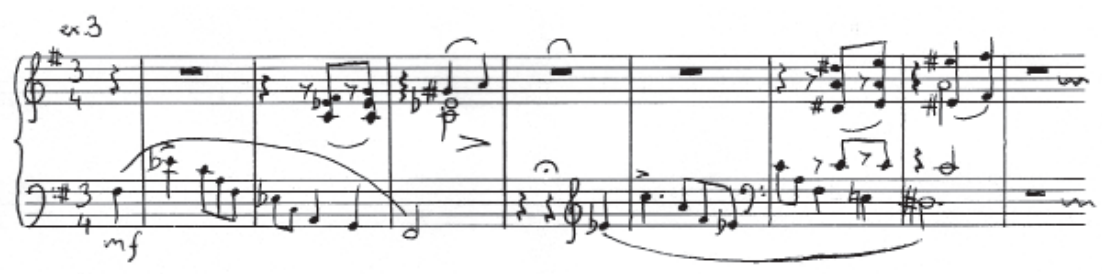

dont le prélude de Tristan et Isolde (1857) est clairement redevable

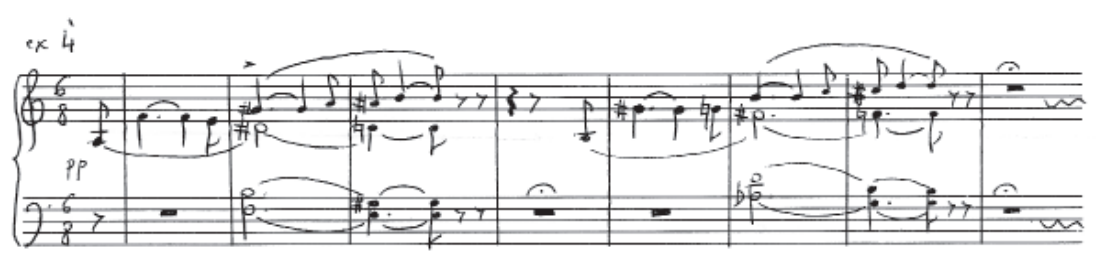

Dans cette seconde thrénodie, Liszt reprend à son tour, en l'inversant, ce dessin accentuato molto 


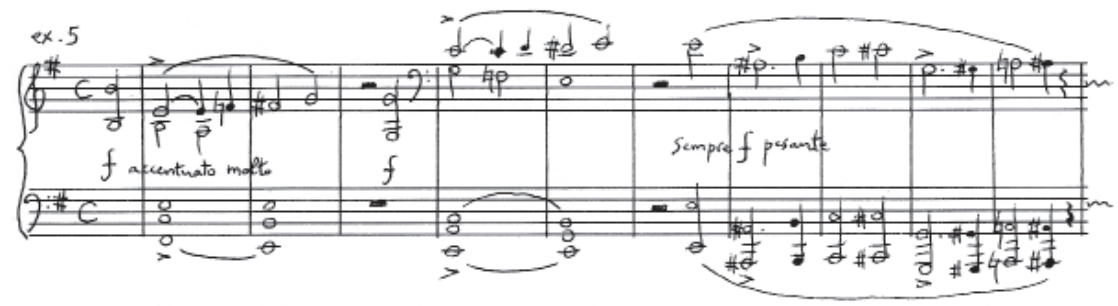

Unissons étranges, silences lourds : déjà l'inquiétude rôde - à moins que ce ne soit la mort - derrière ces accords hachés dans le grave un poco rallentendo, qui trouent à peine le silence (mes. 11-14, puis 26-29), accusant les contrastes de cette première partie $A$ (mes. 1 à 66). Pourtant du médium s'élève une large phrase en octaves, comme une aube nouvelle, éclairant loin, bientôt interrompue (mes. 47 et suivantes) par un hymne grandioso, héroïque, qui lance son défi en octaves puissantes aux deux mains (Liszt écrivait à Marie d'Agoult quarante ans auparavant : " Il nous faut les grandes fautes ou les grandes vertus ! »), pour finalement butter sur le motif souffrant du début... Vanité, tout n'est que vanité...

Mais, dans l'ombre et le silence regagné, s'opère la métamorphose sur l'enharmonie réb-do\# (mes. 67-68). Et voilà que se répand dolce legatissi$m o$ la lumière céleste des arpèges diaphanes qui inondent le clavier de leurs harmonies transparentes tout au long de l'ample partie centrale $B$ (mes. 68 à 161). Aura-t-il ici pensé à l'autre Franz, Schubert, qu'il affectionnait tant ( «Ô génie éternellement jaillissant, génie plein d'amour! Ô cher héros du ciel de ma jeunesse ! ») ? Les premières mesures en tout cas reprennent, à l'enharmonie près, les enchaînements de l'Impromptu opus $90 \mathrm{n}^{\circ} 3$. L'innocence de Schubert, antidote à la passion fatale de Wagner ?... L'espace s'est dilaté et sur ces hauteurs (jusqu'à cinq octaves d'écarts entre basses et chant) plane une phrase ample, sempre dolce e legato, qui déploie ses ailes au long de vingt mesures, sur des ondes de main gauche couvrant la moitié du clavier. Comme une douleur latente néanmoins s'immisce le motif souffrant espressivo dolente ( mes. 96 et suivantes, puis 136 et suivantes) délicatement contrepointé en sixtes chromatiques qui évoquent déjà Fauré. Par trois fois, les grands arpèges vont irradier le clavier.

Le bref $A$ ' nous fait réentendre la phrase de « l'aube nouvelle » émergeant du grave sous un halo de trémolos, dans un climat déjà très Parsifal, avant que n'éclatent de nouveau les octaves héroïques.

Puis, amenant la coda, nouvelle métamorphose plus troublante encore que la première (mes. 207-208) où Liszt combine les éléments de $A$ et $B$ en un symbolisme évident. Émergeant des vagues d'arpèges apaisés, le motif de Tristan, épuré, s'avance, seul, délesté de tous ses nœuds harmoniques 
jusqu'au récitatif monodique, mes. 222 et suivantes. C'est la passion transfigurée et la rédemption par l'amour, chère aux romantiques. Une pluie de grâces se répand sur le clavier tout au long de la dernière page à l'issue de laquelle ne subsiste que le motif mélodique initial, métamorphosé en majeur, lancé seul face à l'Eternité... Fin ouverte s'il en est. Il fallait oser terminer ainsi.

Cette pièce puissante, chant de l'amour et de la mort, semble faire écho à ce qu'il écrivait à sa mère à l'âge de vingt ans : « La vie terrestre n'est qu'une maladie de l'âme, une excitation que les passions entretiennent. L'état naturel de l'âme c'est la quiétude. »

$\mathrm{Si}$, comme l'avait prévu leur auteur, les Cyprès sont rarement joués, les Jeux d'eau à la Villa d'Este n'ont pas tardé, quant à eux, à devenir un des morceaux les plus appréciés du répertoire lisztien. Il faut dire qu'ils sont d'humeur plus souriante et que la virtuosité y déploie ses prestiges et ses séductions de manière plus voyante. Une virtuosité non tonitruante, légère, fluide et scintillante, au service de ce que l'on a pu qualifier de premier morceau impressionniste de l'histoire du piano, à l'origine de toutes les fontaines sonores à venir ( Debussy, Ravel, Respighi...). Car là l'intention descriptive est manifeste. Du début à la fin ce ne sont que jets d'eau, gouttelettes, miroitements, éclaboussures. Description certes, mais pas seulement ; une approche plus approfondie montre qu'on aurait tort de s'arrêter au seul aspect illustratif.

Pour commencer, deux pages et demi d'effets liquides : accords de neuvième en jets d'eau délicatement égrenés, gargouillis de sixtes piquantes, trilles et trémolos multiples faisant scintiller le haut du clavier. Puis, sous l'élément liquide toujours présent, apparaissent des mélodies, dans le médium chantant, généralement à la main gauche, en inversion des paramètres habituels qui veulent que la droite chante sur un accompagnement de main gauche. Car ici les gouttelettes décoratives ne sauraient luire que dans l'aigu de la main droite, réservant à la gauche le chant du médium. Ces mélodies, semblant s'engendrer l'une l'autre, évoluent progressivement du plus simple au plus développé : d'abord un simple motif, sorte de carillon serein et rayonnant dans la clarté de fa\# majeur, la tonalité "séraphique" de Liszt. (thème $1 a$ )

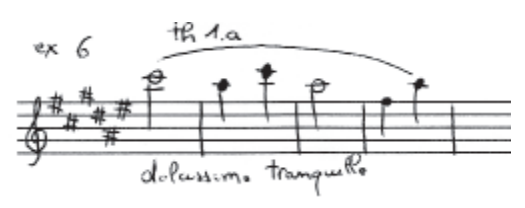

auquel s'enchaîne immédiatement un balancement de main gauche poco espressivo (thème $1 b$ ) 


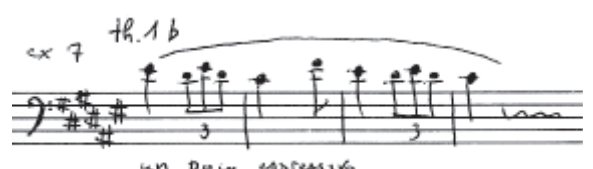

qui se déploie bientôt en une longue phrase épanouie, toute gonflée de sève et d'amour. (thème $1 c$ )

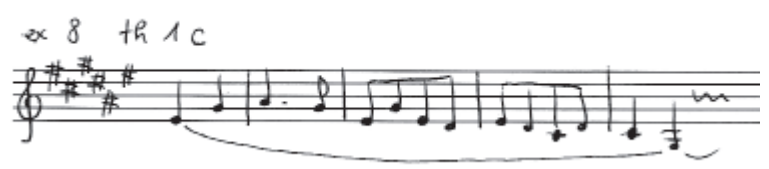

Un peu plus loin apparaît un motif encore plus ouvert, sur sa septième mélodique ascendante (thème 2)

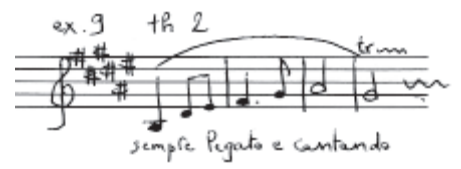

Ainsi, au fil des pages, la main gauche chante-t-elle généreusement, quoique sans pathos. "Lyrisme sans épilepsie » aurait pu dire le jeune Debussy, qui entendit pour la première fois l'œuvre sous les doigts du vieux Liszt lui-même et en fut «stupéfait », au dire de Busoni.

Variantes diverses pour aboutir à un rinforzando aveuglant (mes. 128), cependant que la main droite multiplie les artifices liquides : trémolos divers, staccatos de tierces, arpèges lancés...

L'accalmie toute ruisselante de notes répétées, cristallines, nous conduit au détour d'une modulation attendrie au cœur de l'œuvre : douceur enveloppante des arpèges de main gauche sur lesquels se répandent en accords sereins les différents éléments du thème 1 , soulignés, chose rare, par une citation évangélique : Sed aqua quam ego dabo ei, fiet in eo fons aquae salientis in vitam aeternam (Jean 4,14), c'est-à-dire, aux variantes de traduction près : « Mais l'eau que je lui donnerai deviendra en lui une source d'eau qui jaillira jusqu'à la vie éternelle », tirée du dialogue fameux du Christ et de la Samaritaine. Car cette eau, source d'émerveillement visuel, tactile et sonore est aussi pour Liszt source de vie, en abondance, eau du baptême, image de pureté et d'éternité. Et c'est bien vers l'In- 
fini que s'ouvre la grande amplification du thème $1 c$ (mes. 164-181), culminant en un embrassement complet du clavier rinforzando (mes. 172).

Retour sur terre après la vision béatifique, avec un magnifique développement canonique du thème 2 qui s'enfle peu à peu. La pièce s'achemine alors vers son apothéose sonore (mes. 220) : retour glorieux, à trois reprises, des arpèges de neuvième initiaux en éclaboussures débordantes ff brioso, qui alternent avec l'affirmation monodique, $f f$ elle aussi, du thème 2 . Là, dans la plénitude sonore, les ressources vibrantes de l'instrument sont totalement mises à contribution. Ces résonances puissantes rejoignent peu à peu les régions éthérées de l'aigu (mes. 238-244), vers une dernière page qui s'éloigne toute frémissante de lumière : sous les trémolos scintillants du suraigu se balance doucement le carillon en accords arpégés qui parcourent l'espace par modulations de tierces descendantes, pour se poser finalement sur les accords bienheureux des dernières mesures : tout est accompli.

Accomplissement artistique aussi dans cette musique où rien ne sent le métier ou l'artifice ; tout y est parfaitement original et inventé, que ce soit l'abondance et la découpe mélodique, la variété des textures sonores, une harmonie somptueuse, libérée des pesantes résolutions habituelles, la forme ouverte, en évolution permanente ( $\ll$ je demande simplement le droit de déterminer la forme en fonction du contenu »), sans parler de l'écriture pianistique rutilante.

On appréciera aussi, particulièrement émouvant sous la plume d'un virtuose qui connut durant sa carrière les plus délirants succès d'estrade, le refus de tout effet facile, une parfaite économie de moyens où chaque note compte. Rien de flatteur dans cette musique sans concession qui exige de l'auditeur une écoute (et une réécoute) attentive et concentrée, mais dont il sera récompensé au centuple.

Et, ayant évoqué ici ou là l'expressionnisme, le symbolisme ou l'impressionnisme, comment ne pas s'incliner devant l'extraordinaire modernité du vieux Liszt qui, sans jamais se répéter, semble avoir anticipé toutes les évolutions futures de la musique. Et comment ne pas remercier Hippolyte d'avoir (involontairement...) suscité ces merveilles. Les grands cyprès sont toujours là, les jeux d'eau aussi, indifférents peut être aux souffrances humaines, et qui nous disent la pérennité de la vie et le bonheur d'exister.

\section{Notes}

Note : Toutes les citations indiquées entre guillemets sont extraites de la vaste correspondance de Liszt. Voir, en particulier : Correspondance de Liszt et de Madame d'Agoult 1840-1864, Fayard, 2001, 1344 p. ; Correspondance Liszt-Richard Wagner, Gallimard, 2007, 1344 p. 